\title{
Preoperative Fibrinogen-Albumin Ratio, Potential Prognostic Factors for Bladder Cancer Patients Undergoing Radical Cystectomy: A Two-Center Study
}

This article was published in the following Dove Press journal:

Cancer Management and Research

\author{
Jiangang Chen (iD) ${ }^{1,2}$ \\ Lin $\mathrm{HaO}^{1,3}$ \\ Shaoqi Zhang $\mathbb{B}^{\prime}$ \\ Yong Zhang ${ }^{2}$ \\ Bingzheng Dong ${ }^{1,3}$ \\ Qianjin Zhang' \\ Conghui Han (1D) 1,3
}

'Medical College of Soochow University, Suzhou, Jiangsu Province, 215123, People's Republic of China; ${ }^{2}$ Department of Urology, The Second Affiliated Hospital of Nantong University, Nantong, Jiangsu Province, 22600I, People's Republic of China; ${ }^{3}$ Department of Urology, Xuzhou Central Hospital, Xuzhou Clinical College Affiliated to Xuzhou Medical University, Xuzhou, Jiangsu Province, 221009, People's Republic of China
Correspondence: Conghui Han Department of Urology, Xuzhou Central Hospital, Xuzhou Clinical College Affiliated to Xuzhou Medical University, 199 Jiefang South Road, Xuzhou, Jiangsu, 221009, People's Republic of China Email conghuihandoctor@163.com
Background: We conducted a two-center study to investigate the prognostic value of preoperative fibrinogen-albumin ratio (FAR) in patients undergoing radical cystectomy (RC).

Methods: The clinical and survival data of 267 patients with bladder cancer $(\mathrm{BCa})$ treated with RC were collected, of which 140 patients from Xuzhou Central Hospital were divided into training set and 127 patients from The Second Affiliated Hospital of Nantong University were divided into validation set. X-tile software was used to obtain the optimal cut-off values for preoperative platelet-to-lymphocyte ratio (PLR), neutrophil-to-lymphocyte ratio (NLR) and FAR. Receiver operating characteristic (ROC) curves were used to compare the predictive ability of PLR, NLR, FAR and modified Glasgow prognostic score (mGPS). Kaplan-Meier curves were used to assess overall survival (OS) and progression-free survival (PFS) of patients in different FAR groups. Univariate and multivariate Cox regression were used to assess patients' independent risk factors, and $\mathrm{R}$ software was used to construct prognostic nomograms.

Results: In the training set, the optimal cut-off values for PLR, NLR and FAR were 76.76, 3.97 and 0.08 , respectively. Both in the training and validation sets, FAR had better ability to predict OS and PFS than PLR and NLR, and patients in the higher FAR group had worse OS and PFS. In the multivariate Cox regression analysis, FAR was an independent risk factor for OS [hazard ratio (HR) 3.569, 95\% confidence interval (CI): $1.015-12.546, \mathrm{P}=0.047$ ] and PFS [HR 5.071, 95\% CI: 1.394-18.451, $\mathrm{P}=0.014$ ]. In addition, FAR-based prognostic nomograms had high predictive ability than TNM staging.

Conclusion: Preoperative FAR is an independent prognostic factor for OS and PFS in $\mathrm{BCa}$ patients treated with $\mathrm{RC}$, and a high FAR predicted a poor prognosis. In addition, a prognostic nomogram based on FAR can better predict individual survival.

Keywords: fibrinogen-albumin ratio, bladder cancer, radical cystectomy, overall survival, progression-free survival

\section{Introduction}

Bladder cancer $(\mathrm{BCa})$, as a common malignancy of the genitourinary system worldwide, has an increasing morbidity and mortality rate. ${ }^{1}$ According to the degree of invasion, $\mathrm{BCa}$ can be divided into non-muscle invasive bladder cancer (NMIBC) and muscle invasive bladder cancer (MIBC). ${ }^{2}$ For early stage and NMIBC, one can choose the treatment of preserving the bladder. Once the tumor invades the muscle layer and progresses to MIBC 
or recurs repeatedly, radical cystectomy (RC) and urinary diversion is required, but the prognosis after $\mathrm{RC}$ is still poor, with a 5-year survival rate of only $50 \%$. $^{3,4}$ Therefore, there is an urgent need for a simple and accurate clinical biomarker for early detection and identification of BCa patients with disease progression or poor prognosis.

The hypercoagulable state of the blood is associated with malignancy. ${ }^{5}$ Fibrinogen is a kind of glycoprotein synthesized by hepatocytes, which can be converted into fibrin and plays an important physiological role in the coagulation process. ${ }^{6}$ Albumin is also produced by the liver, with anti-inflammatory, antioxidant activity and prevent apoptosis, and serum albumin is a key indicator of nutritional status. ${ }^{7,8}$ Preoperative fibrinogen-albumin ratio (FAR), as a new biomarker, combined with blood nutritional status and coagulation, has been widely used as a predictor of the prognosis of various malignancies. ${ }^{8-10}$ In this study, we investigated the prognostic value of preoperative FAR levels on postoperative outcomes in patients undergoing $\mathrm{RC}$ in a multicenter study and compared it with other established inflammation markers, including platelet-to-lymphocyte ratio (PLR) and neutrophil-to-lymphocyte ratio (NLR).

\section{Patients and Methods}

\section{Patients}

In our study, we retrospectively analyzed 267 patients with BCa who underwent RC at two hospitals: 140 patients from the Xuzhou Central Hospital from January 2015 to June 2019 and 127 patients from the Second Affiliated Hospital of Nantong University from March 2009 to October 2018. According to the 2002 American Joint Committee on Cancer/Union for International Cancer Control TNM classification, all patients were pathologically diagnosed with $\mathrm{BCa}{ }^{11}$ The methodology of this study followed the criteria outlined in the Declaration of Helsinki (as revised in 2013) and was ethically approved by the Ethics Committees and Institutional Review Boards of all participating institutions. ${ }^{12}$ Written informed consent was provided by all patients or their relatives who participated in this study.

The inclusion criteria were as follows: a. pathological diagnosis after transurethral resection of bladder tumor (TURBt) was MIBC and high-grade $\mathrm{T} 1 \mathrm{BCa}$; b. patients underwent $\mathrm{RC}$; c. more than 18 years of age. And the exclusion criteria included the following: a. patients with coexisting other malignancies; b. preoperative chemotherapy or radiotherapy; c. patients with incomplete or missing followup information; d. patients with other disease-related conditions that significantly affected survival time.

\section{Variables and Follow-Up}

Patients' blood samples were collected two days before surgery or closest to the time of surgery. Patient

Table I Baseline Characteristics of the Study Population

\begin{tabular}{|c|c|c|c|c|}
\hline \multirow[t]{2}{*}{ Characteristic } & $\begin{array}{c}\text { All } \\
\text { Patients }\end{array}$ & $\begin{array}{c}\text { Training } \\
\text { Set }\end{array}$ & $\begin{array}{c}\text { Validation } \\
\text { Set }\end{array}$ & \multirow[t]{2}{*}{$P$ value } \\
\hline & No. (\%) & No. (\%) & No. (\%) & \\
\hline Total patients & 267 & 140 & 127 & \\
\hline Age, y & & & & 0.670 \\
\hline$\leq 65$ & $134(50.2)$ & $72(51.4)$ & $62(48.8)$ & \\
\hline$>65$ & $133(49.8)$ & $68(48.6)$ & $65(51.2)$ & \\
\hline Gender & & & & 0.832 \\
\hline Male & $230(86.1)$ & $120(85.7)$ & $110(86.6)$ & \\
\hline Female & 37 (13.9) & $20(14.3)$ & $17(13.4)$ & \\
\hline $\mathrm{BMI}, \mathrm{kg} / \mathrm{m}^{2}$ & & & & 0.752 \\
\hline$\leq 24.0$ & $155(58.1)$ & $79(56.4)$ & $75(59.1)$ & \\
\hline$>24.0$ & $112(41.9)$ & $61(43.6)$ & $52(40.9)$ & \\
\hline T-stage & & & & 0.016 \\
\hline TI-2 & 132 (49.4) & $79(56.4)$ & $53(41.7)$ & \\
\hline T3-4 & $135(50.6)$ & 61 (43.6) & $74(58.3)$ & \\
\hline $\mathrm{N}$-stage & & & & 0.031 \\
\hline No & $224(83.9)$ & III (79.3) & II3 (89.0) & \\
\hline $\mathrm{N}+$ & $43(16.1)$ & $29(20.7)$ & $14(11.0)$ & \\
\hline M-stage & & & & 0.863 \\
\hline Mo & $255(95.5)$ & $134(95.7)$ & $12 \mid(95.3)$ & \\
\hline MI & $12(4.5)$ & $6(4.3)$ & $6(4.7)$ & \\
\hline PLR & & & & 0.395 \\
\hline$\leq 76.76$ & $4 \mathrm{I}(15.4)$ & $24(17.1)$ & $17(13.4)$ & \\
\hline$>76.76$ & $226(84.6)$ & $116(82.9)$ & $110(86.6)$ & \\
\hline NLR & & & & 0.252 \\
\hline$\leq 3.97$ & $180(67.4)$ & $90(64.3)$ & $90(70.9)$ & \\
\hline$>3.97$ & $87(32.6)$ & $50(35.7)$ & $37(29.1)$ & \\
\hline FAR & & & & 0.178 \\
\hline$\leq 0.08$ & $174(65.2)$ & $86(61.4)$ & $88(69.3)$ & \\
\hline$>0.08$ & $93(34.8)$ & $54(38.6)$ & $39(30.7)$ & \\
\hline mGPS & & & & 0.056 \\
\hline 0 & $193(72.3)$ & $93(66.4)$ & $100(78.7)$ & \\
\hline 1 & $46(17.2)$ & $31(22.1)$ & $15(11.8)$ & \\
\hline 2 & $28(10.5)$ & $16(11.4)$ & $12(9.4)$ & \\
\hline Hemoglobin, g/L & & & & 0.899 \\
\hline$\leq 110$ & $64(24.0)$ & $34(24.3)$ & $30(23.6)$ & \\
\hline$>110$ & $203(76.0)$ & $106(75.7)$ & $97(76.4)$ & \\
\hline
\end{tabular}

Abbreviations: BMI, body mass index; PLR, platelet-lymphocyte ratio; NLR, neutrophil-lymphocyte ratio; FAR, fibrinogen-albumin ratio. 
demographic information was downloaded from the hospital's electronic medical records and collected by trained personnel, including age ( $\leq 65$ years and $>65$ years), gender (male and female), body mass index (BMI) $\left(\leq 24.0 \mathrm{~kg} / \mathrm{m}^{2}\right.$ and $\left.>24.0 \mathrm{~kg} / \mathrm{m}^{2}\right)$, T-stage (T1-2 and T34), N-stage (N0 and N+), M-stage (M0 and M1), fibrinogen, albumin, C-reactive protein, platelet, lymphocyte, neutrophil and hemoglobin. Outpatient follow-up or telephone interviews were conducted periodically by dedicated personnel on all postoperative patients, and the survival status or cause of death was collected in each

A

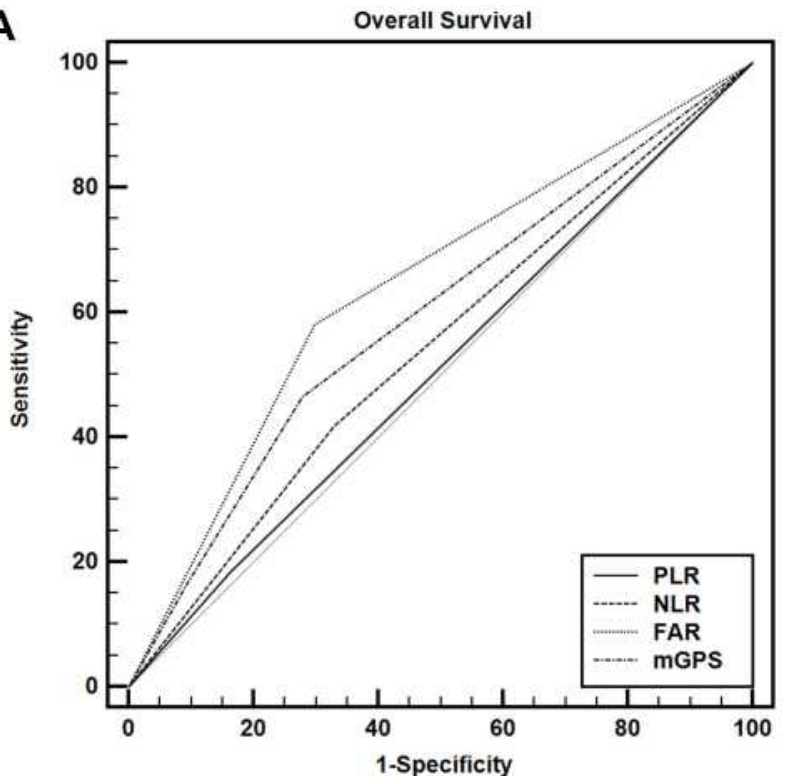

C

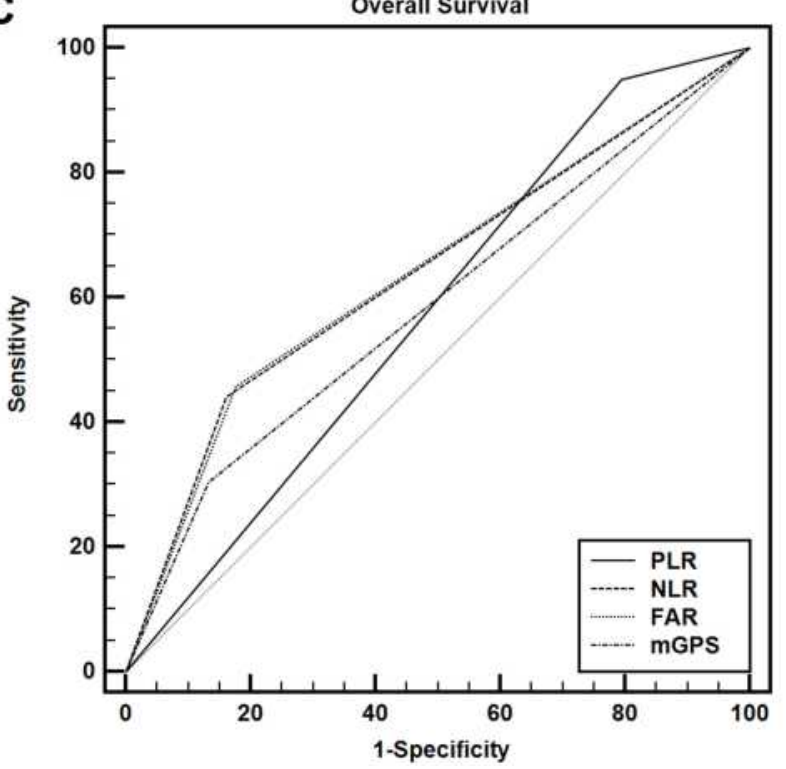

follow-up visit. Overall survival (OS) time was the time between the completion of surgery and death from all causes. Progression-free survival (PFS) refers to the time between completion of surgery to disease progression or the occurrence of death.

\section{Statistical Analysis}

PLR, NLR and FAR were calculated as follows: absolute number of platelets divided by absolute number of lymphocytes, absolute number of neutrophils divided by absolute number of lymphocytes, and fibrinogen divided by albumin.
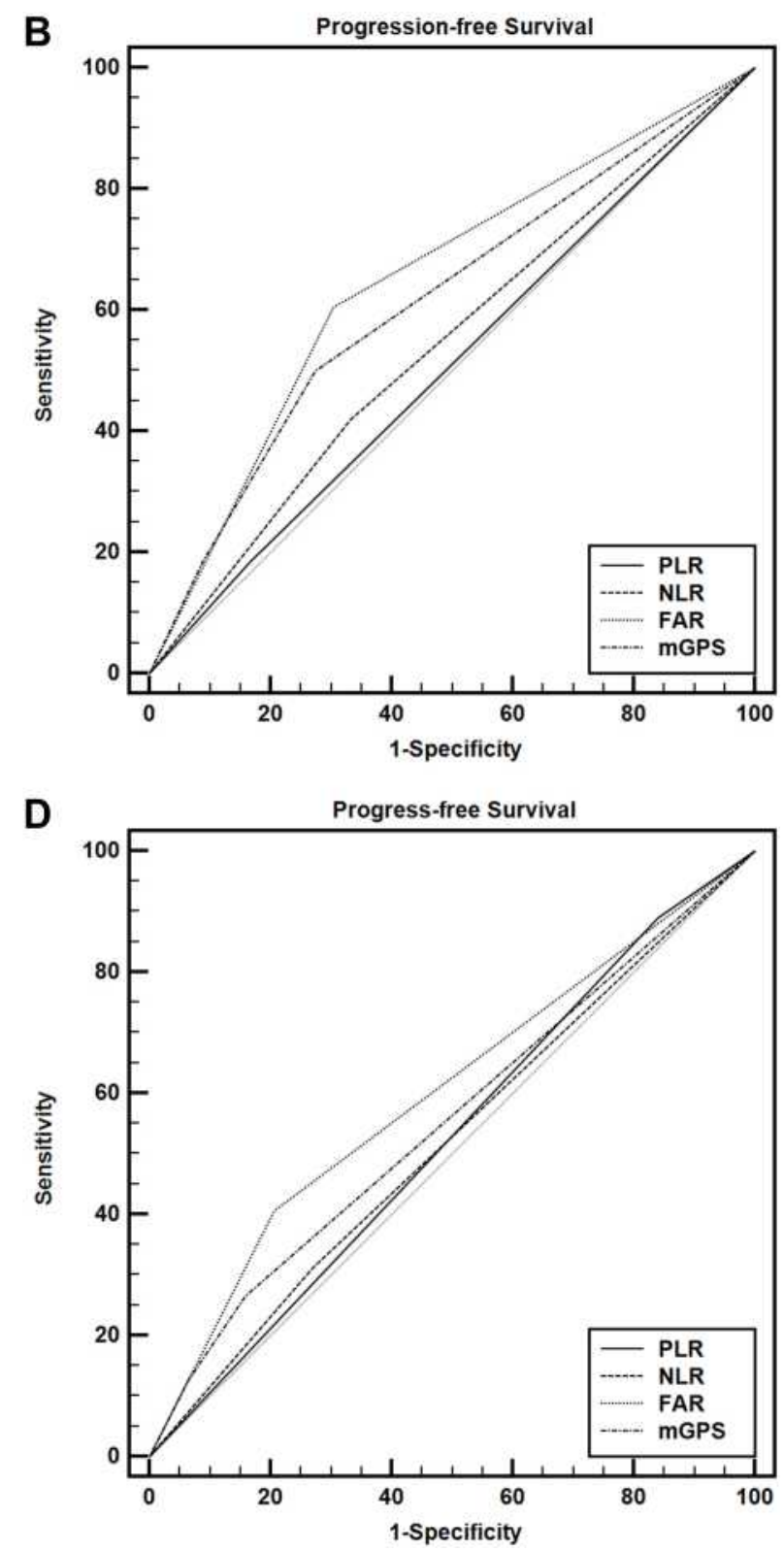

Figure I Comparison of receiver operating characteristic (ROC) curves of overall survival (OS) and progression-free survival (PFS) between fibrinogen-albumin ratio (FAR), platelet-lymphocyte ratio (PLR), neutrophil-lymphocyte ratio (NLR) and modified Glasgow prognostic score ( $m G P S)$ in training and validation sets. (A) OS in training set; (B) PFS in training set; (C) OS in validation set; (D) PFS in validation set. 
The modified Glasgow prognostic score (mGPS) was computed as previously described. ${ }^{13} \mathrm{X}$-tile software (Version 3.6.1, Yale University) was used to obtain the optimal cut-off values for preoperative PLR, NLR and FAR. One hundred and forty patients from the Zhongda Hospital were divided into training set and 127 patients from the Second Affiliated Hospital of Nantong University were divided into validation set. Classified variables were represented by percentages and numbers. Pearson's test or Fisher exact test was used to test for differences in categorical variables. Receiver operating characteristic (ROC) curves were used to compare the predictive ability of PLR, NLR and FAR and expressed as the area under the curve (AUC). OS and PFS survival curves were plotted using the Kaplan-Meier curve and evaluated using the Log-rank test. In the training set, univariate and multivariate Cox proportional hazard model was used to evaluate the association variables with OS and PFS, and hazard ratios (HR) and 95\% confidence interval (CI) were calculated. Based on the multivariate Cox regression result, the $\mathrm{R}$ software was used to construct prognostic nomograms for OS and PFS, and the consistency index (C-index), ROC curves, and calibration curves were used to evaluate the predictive ability of the nomograms and validated in the validation set. All statistical analyses were performed using SPSS software (Version 24.0, IBM Corp) and RStudio software (Version 1.2.5033, JJ Allaire Corp). All statistical tests were considered statistically significant at $\mathrm{P}<0.05$.

\section{Results}

In the training set, the optimal cut-off values for PLR, NLR and FAR were 76.76, 3.97 and 0.08 , respectively (Figure S1). The demographic and clinicopathological characteristics of the study population are presented in Table 1 . We observed statistically significant differences between the two sets in $\mathrm{T}$ stage and $\mathrm{N}$ stage. We found that both in the training set or validation set, the majority of patients were male (training set: $85.7 \%$; validation set: $86.6 \%$ ), N0 stage (training set: 79.3\%; validation set: $89.0 \%$ ), M0 stage (training set: $95.7 \%$; validation set: $95.3 \%$ ), PLR >76.76 (training set: $82.9 \%$; validation set: $86.6 \%$ ), NLR $\leq 3.97$ (training set: $64.3 \%$; validation set: $70.9 \%$ ), FAR $\leq 0.08$ (training set: $61.4 \%$; validation set: $69.3 \%$ ), mGPS=0 (training set: $66.4 \%$; validation set: $78.7 \%$ ) and hemoglobin $>110$ (training set: $75.7 \%$; validation set: 76.4\%). The ROC curves showed that both in the training and validation sets, FAR had better ability to predict OS (training set: FAR $\mathrm{AUC}=0.641$; validation set: $\mathrm{FAR}$ $\mathrm{AUC}=0.641$ ) and PFS (training set: $\mathrm{FAR} \mathrm{AUC}=0.651$; validation set: FAR $\mathrm{AUC}=0.600)$ than PLR, NLR and mGPS (Figure 1 and Table 2).

Based on the cut-off value of FAR, patients were divided into high FAR $(>0.08)$ group and low FAR $(\leq 0.08)$ group. Baseline characteristics of all patients are shown in Table 3 when using FAR as an assessment tool. Chi-square test showed that FAR was associated with gender, $\mathrm{T}$ stage, $\mathrm{N}$ stage, NLR, mGPS, and hemoglobin (All $\mathrm{P}<0.05)$ in both the training and validation sets. We can find that both in the training and validation sets, patients in the high FAR group had higher age, higher proportion of female, higher T-stage, N-stage, M-stage, higher NLR, higher mGPS and lower hemoglobin compared to the low FAR group (Table 3).

We analyzed the effect of FAR on OS and PFS using Kaplan-Meier curves in the training and validation sets. We

Table 2 Analysis of Predictive Accuracy Through the Evaluation of the Area Under the Curve (AUC)

\begin{tabular}{|c|c|c|c|c|c|c|}
\hline \multirow[t]{2}{*}{ Characteristics } & \multicolumn{3}{|c|}{ Overall Survival } & \multicolumn{3}{|c|}{ Progression-Free Survival } \\
\hline & AUC & 95\% Cl & $P$ value & AUC & $95 \% \mathrm{Cl}$ & $P$ value \\
\hline \multicolumn{7}{|l|}{ Train set } \\
\hline PLR & 0.511 & $0.425-0.596$ & 0.842 & 0.509 & $0.423-0.594$ & 0.873 \\
\hline NLR & 0.544 & $0.458-0.629$ & 0.403 & 0.544 & $0.458-0.628$ & 0.426 \\
\hline FAR & $0.64 I$ & $0.556-0.720$ & 0.008 & 0.651 & $0.566-0.729$ & 0.006 \\
\hline mGPS & 0.594 & $0.508-0.677$ & 0.075 & 0.616 & $0.530-0.697$ & 0.035 \\
\hline \multicolumn{7}{|l|}{ Validation set } \\
\hline PLR & 0.578 & $0.487-0.665$ & 0.133 & 0.525 & $0.434-0.614$ & 0.631 \\
\hline NLR & 0.639 & $0.550-0.723$ & 0.007 & 0.521 & $0.43 \mathrm{I}-0.6 \mathrm{II}$ & 0.678 \\
\hline FAR & 0.641 & $0.55 \mathrm{I}-0.724$ & 0.006 & 0.600 & $0.509-0.686$ & 0.052 \\
\hline mGPS & 0.586 & $0.496-0.673$ & 0.094 & 0.555 & $0.464-0.643$ & 0.285 \\
\hline
\end{tabular}

Note: $P$-value $<0.05$ are shown in bold.

Abbreviations: AUC, area under the curve; Cl, confidence interval; PLR, platelet-lymphocyte ratio; PDM, platelet $\times$ D-dimer; NLFgA, neutrophil $\times$ Fibrinogen/ (lymphocyte $\times$ albumin); NLDA, neutrophil $\times$ D-dimer/(lymphocyte $\times$ albumin). 
Table 3 Baseline Characteristics of All Patients When Using FAR as an Assessment Tool

\begin{tabular}{|c|c|c|c|c|c|c|c|c|}
\hline \multirow[t]{3}{*}{ Characteristic } & \multicolumn{4}{|c|}{ Train Set $(N=\mid 40)$} & \multicolumn{4}{|c|}{ Validation Set $(N=\mid 27)$} \\
\hline & \multirow{2}{*}{$\begin{array}{c}\text { FAR } \leq 0.08 \\
\text { No. (\%) }\end{array}$} & \multirow{2}{*}{$\begin{array}{c}\text { FAR }>0.08 \\
\text { No. }(\%)\end{array}$} & \multirow[t]{2}{*}{$\chi^{2}$} & \multirow[t]{2}{*}{$P$ value } & \multirow{2}{*}{$\begin{array}{c}\text { FAR } \leq 0.08 \\
\text { No. (\%) }\end{array}$} & \multirow{2}{*}{$\begin{array}{c}\text { FAR }>0.08 \\
\text { No. (\%) }\end{array}$} & \multirow[t]{2}{*}{$\chi^{2}$} & \multirow[t]{2}{*}{$P$ value } \\
\hline & & & & & & & & \\
\hline Total patients & $86(61.4)$ & $54(38.6)$ & & & $88(69.3)$ & $39(30.7)$ & & \\
\hline Age, y & & & 7.289 & 0.007 & & & 0.160 & 0.689 \\
\hline$\leq 65$ & $52(60.5)$ & $20(37.0)$ & & & $44(50.0)$ & $18(46.2)$ & & \\
\hline$>65$ & $34(39.5)$ & $34(63.0)$ & & & $44(50.0)$ & $21(53.8)$ & & \\
\hline Gender & & & 4.522 & 0.033 & & & 4.559 & 0.033 \\
\hline Male & $78(90.7)$ & $42(77.8)$ & & & $80(90.9)$ & 30 (76.9) & & \\
\hline Female & $8(9.3)$ & $12(22.2)$ & & & $8(9.1)$ & $9(23.1)$ & & \\
\hline BMI, $\mathrm{kg} / \mathrm{m}^{2}$ & & & 4.645 & 0.031 & & & 2.410 & 0.121 \\
\hline$\leq 24.0$ & $43(50.0)$ & $37(68.5)$ & & & $48(54.5)$ & $27(69.2)$ & & \\
\hline$>24.0$ & $43(50.0)$ & $17(3 \mid .5)$ & & & $40(45.5)$ & $12(30.8)$ & & \\
\hline T-stage & & & 8.799 & 0.003 & & & 5.993 & 0.014 \\
\hline TI-2 & $57(66.3)$ & $22(40.7)$ & & & $43(48.9)$ & $10(25.6)$ & & \\
\hline T3-4 & $29(33.7)$ & $32(59.3)$ & & & $45(51.1)$ & $29(74.4)$ & & \\
\hline $\mathrm{N}$-stage & & & 6.205 & 0.013 & & & 5.167 & 0.023 \\
\hline No & $74(86.0)$ & $37(68.5)$ & & & $82(93.2)$ & $31(79.5)$ & & \\
\hline NI-2 & $12(14.0)$ & I7 (3|.5) & & & $6(6.8)$ & $8(20.5)$ & & \\
\hline M-stage & & & 0.346 & 0.557 & & & 8.196 & 0.004 \\
\hline Mo & $83(96.5)$ & $51(94.4)$ & & & 87 (98.9) & 34 (87.2) & & \\
\hline MI & $3(3.5)$ & $3(5.6)$ & & & I (I.I) & $5(12.8)$ & & \\
\hline PLR & & & 20.146 & $<0.001$ & & & 0.016 & 0.901 \\
\hline$\leq 76.76$ & $5(5.8)$ & $19(35.2)$ & & & $12(13.6)$ & $5(12.8)$ & & \\
\hline$>76.76$ & 81 (94.2) & $35(64.8)$ & & & $76(86.4)$ & 34 (87.2) & & \\
\hline NLR & & & 15.073 & $<0.001$ & & & 20.283 & $<0.001$ \\
\hline$\leq 3.97$ & $66(76.7)$ & $24(44.4)$ & & & $73(83.0)$ & $17(43.6)$ & & \\
\hline$>3.97$ & $20(23.3)$ & $30(55.6)$ & & & I5 (I7.0) & $22(56.4)$ & & \\
\hline mGPS & & & 40.217 & $<0.001$ & & & 7.239 & 0.027 \\
\hline 0 & $74(86.0)$ & $19(35.2)$ & & & $75(85.2)$ & $25(64.1)$ & & \\
\hline 1 & $10(11.6)$ & $21(38.9)$ & & & $7(8.0)$ & $8(20.5)$ & & \\
\hline 2 & $2(2.3)$ & 14 (25.9) & & & $6(6.8)$ & $6(15.4)$ & & \\
\hline Hemoglobin, $g / L$ & & & 19.428 & $<0.001$ & & & 15.838 & $<0.001$ \\
\hline$\leq 110$ & $10(11.6)$ & $24(44.4)$ & & & $12(13.6)$ & $18(46.2)$ & & \\
\hline$>110$ & $76(88.4)$ & $30(55.6)$ & & & $76(86.4)$ & $21(53.8)$ & & \\
\hline
\end{tabular}

Abbreviations: BMI, body mass index; PLR, platelet-lymphocyte ratio; NLR, neutrophil-lymphocyte ratio; FAR, fibrinogen-albumin ratio.

found that FAR was associated with OS and PFS and patients in the higher FAR group had worse OS (Figure 2A and C) and PFS (Figure 2B and D). In the training set, we performed univariate and multivariate Cox regression to find independent risk factors for OS and PFS in patients with $\mathrm{BCa}$ (Table 4). We found that TNM stage and FAR were independent risk factors for $\mathrm{BCa}$ patients, and higher FAR was associated with poorer prognosis in BCa patients (OS: HR, 2.031, 95\%
$\mathrm{CI}=1.071-3.851 ; \mathrm{p}=0.030 ;$ PFS: HR, 2.330, 95\% $\mathrm{CI}=1.160-4.680 ; \mathrm{p}=0.017)$. Based on the above multivariate Cox regression results, we constructed prognostic nomograms of OS and PFS that included TNM stage and FAR (Figure 3). We performed ROC curve analysis for OS and PFS at 3 and 5 years, and found high AUC (All >0.7) in the training and validation sets (Figures 4 and $\underline{\mathrm{S}}$ ). In addition, the ROC curves showed that the FAR-based prognostic nomogram had higher 


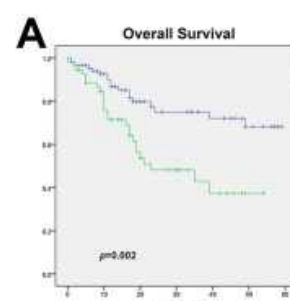

A1 matom

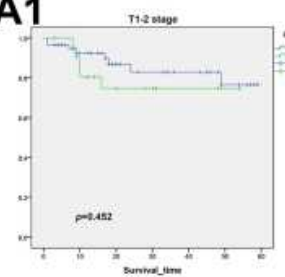

A2

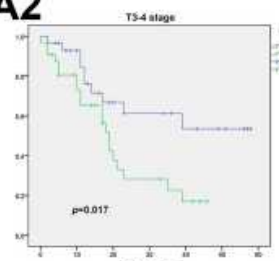

A3

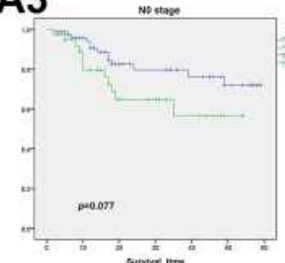

A4

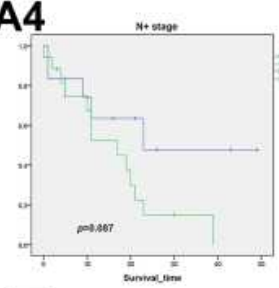

A5

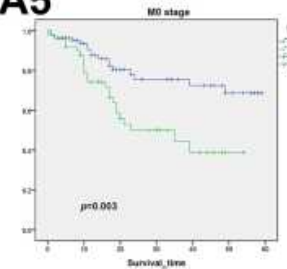

A6

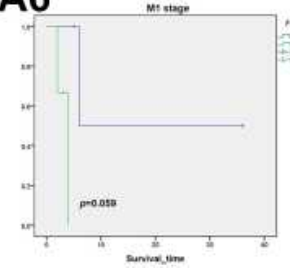

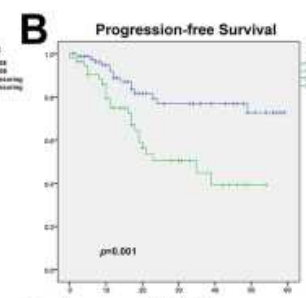

B1

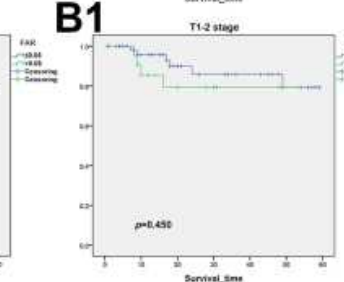

B2

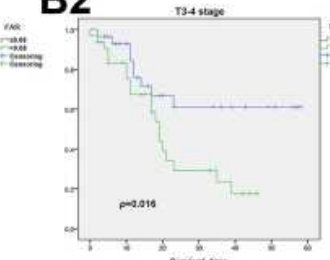

B3

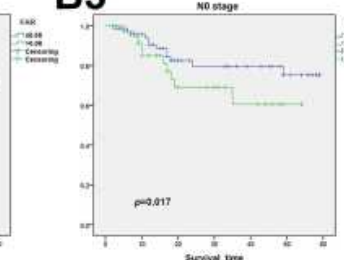

B4

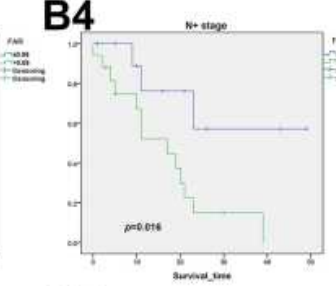

B5

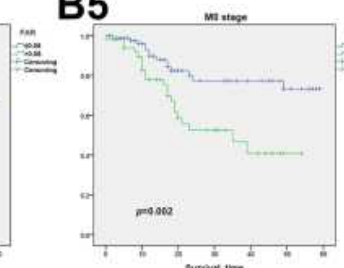

B6

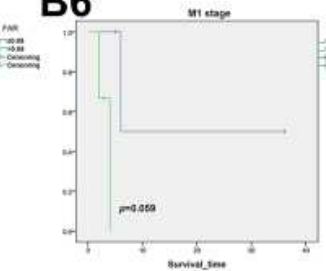

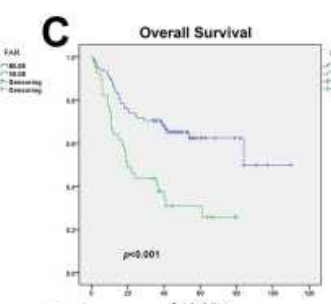

C1 risure

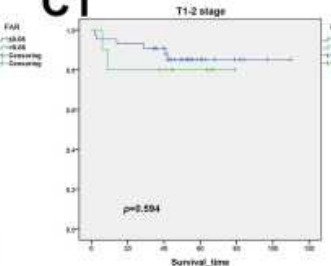

C2

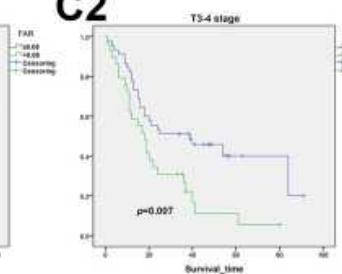

C3

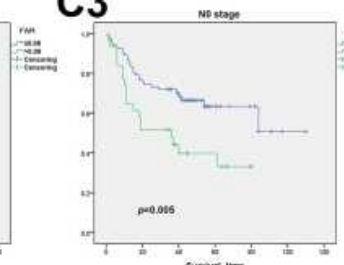

C4
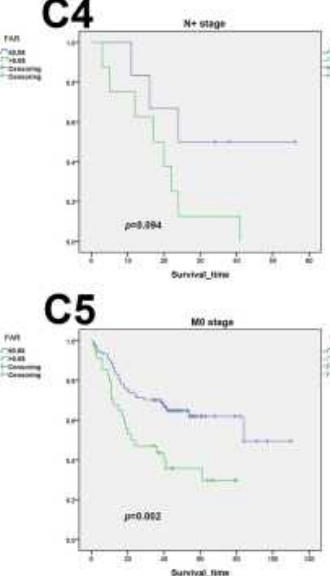

C6

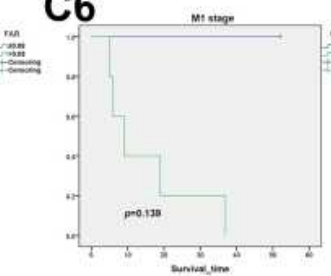

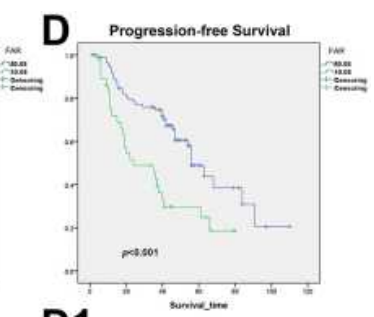

D1 ristame

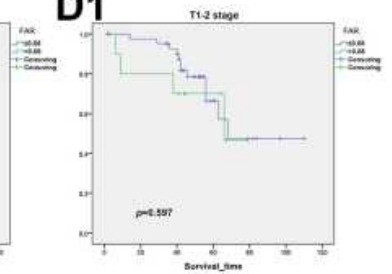

D2

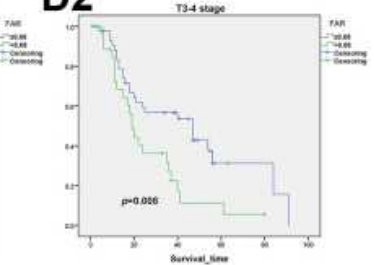

D3

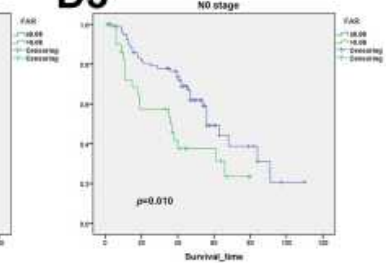

D4
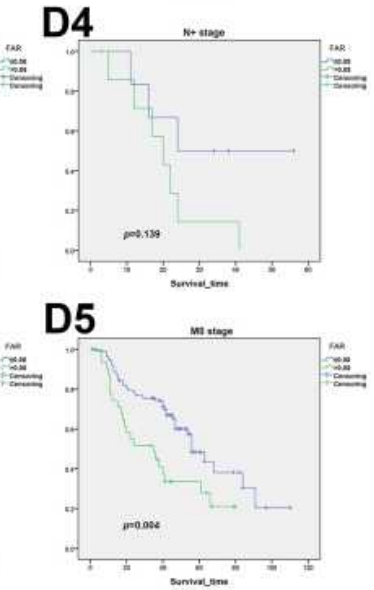

D6

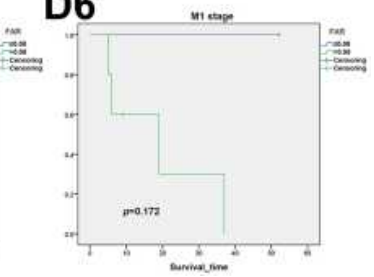

Figure 2 Kaplan-Meier curves of overall survival (OS) and progression-free survival (PFS) based on fibrinogen-albumin ratio (FAR) value in training and validation sets. (A) OS curve in training set; (B) PFS curve in training set; (C) OS curve in validation set; (D) PFS curve in validation set. 
Table 4 Univariate and Multivariate Analyses of Factors Associated with Overall Survival (OS) and Progression-Free Survival (PFS) in Train Set

\begin{tabular}{|c|c|c|c|c|c|c|c|c|}
\hline \multirow[t]{3}{*}{ Characteristics } & \multicolumn{4}{|l|}{ Overall Survival } & \multicolumn{4}{|c|}{ Progression-Free Survival } \\
\hline & \multicolumn{2}{|l|}{ Univariate Analyses } & \multicolumn{2}{|c|}{ Multivariate Analyses } & \multicolumn{2}{|l|}{ Univariate Analyses } & \multicolumn{2}{|c|}{ Multivariate Analyses } \\
\hline & HR (95\% Cl) & $P$ value & HR (95\% CI) & $P$ value & HR (95\% Cl) & $P$ value & HR $(95 \% \mathrm{Cl})$ & $P$ value \\
\hline \multicolumn{9}{|l|}{ Age, y } \\
\hline$\leq 65$ & Reference & & Reference & & Reference & & Reference & \\
\hline$>65$ & $1.292(0.708-2.358)$ & 0.404 & - & 0.225 & $1.235(0.65 I-2.34 I)$ & 0.518 & - & 0.310 \\
\hline \multicolumn{9}{|l|}{ Gender } \\
\hline Male & Reference & & Reference & & Reference & & Reference & \\
\hline Female & $1.226(0.553-2.806)$ & 0.596 & - & 0.976 & $1.224(0.510-2.934)$ & 0.651 & - & 0.921 \\
\hline \multicolumn{9}{|l|}{$\mathrm{BMI}, \mathrm{kg} / \mathrm{m}^{2}$} \\
\hline$\leq 24.0$ & Reference & & Reference & & Reference & & Reference & \\
\hline$>24.0$ & $0.938(0.509-1.731)$ & 0.838 & - & 0.780 & $0.845(0.437-1.635)$ & 0.616 & - & 0.886 \\
\hline \multicolumn{9}{|l|}{ T-stage } \\
\hline TI-2 & Reference & & Reference & & Reference & & Reference & \\
\hline T3-4 & $3.518(1.833-6.755)$ & $<0.001$ & $2.188(1.070-4.474)$ & 0.023 & $4.320(2.095-8.908)$ & $<0.001$ & $2.630(1.194-5.792)$ & 0.016 \\
\hline \multicolumn{9}{|l|}{$\mathrm{N}$-stage } \\
\hline No & Reference & & Reference & & Reference & & Reference & \\
\hline $\mathrm{NI}-2$ & $3.924(2.129-7.233)$ & $<0.001$ & $2.408(1.230-4.715)$ & 0.010 & 4.015 (2.097-7.687) & $<0.001$ & $2.324(1.142-4.729)$ & 0.020 \\
\hline \multicolumn{9}{|l|}{ M-stage } \\
\hline Mo & Reference & & Reference & & Reference & & Reference & \\
\hline MI & $4.345(1.319-14.315)$ & 0.016 & $3.569(1.015-12.546)$ & 0.047 & $5.397(1.632-17.850)$ & 0.006 & $5.07 \mid(1.394-18.451)$ & 0.014 \\
\hline \multicolumn{9}{|l|}{ PLR } \\
\hline$\leq 76.76$ & Reference & & Reference & & Reference & & Reference & \\
\hline$>76.76$ & $1.123(0.519-2.430)$ & 0.769 & - & 0.319 & $1.169(0.513-2.660)$ & 0.710 & - & 0.265 \\
\hline \multicolumn{9}{|l|}{ NLR } \\
\hline$\leq 3.97$ & Reference & & Reference & & Reference & & Reference & \\
\hline$>3.97$ & $1.256(0.683-2.309)$ & 0.464 & - & 0.533 & $1.249(0.654-2.385)$ & 0.501 & - & 0.382 \\
\hline \multicolumn{9}{|l|}{ FAR } \\
\hline$\leq 0.08$ & Reference & & Reference & & Reference & & Reference & \\
\hline$>0.08$ & $2.571(1.397-4.734)$ & 0.002 & $2.03 I(I .07 I-3.85 I)$ & 0.030 & $2.842(1.476-5.472)$ & 0.002 & $2.330(1.160-4.680)$ & 0.017 \\
\hline \multicolumn{9}{|l|}{ mGPS } \\
\hline 0 & Reference & & Reference & & Reference & & Reference & \\
\hline 1 & $1.894(0.953-3.767)$ & 0.069 & - & 0.466 & $2.091(1.007-4.340)$ & 0.048 & - & 0.404 \\
\hline 2 & $1.789(0.764-4.186)$ & 0.180 & - & 0.269 & $2.133(0.893-5.097)$ & 0.088 & - & 0.362 \\
\hline \multicolumn{9}{|l|}{ Hemoglobin, g/L } \\
\hline$\leq 110$ & Reference & & Reference & & Reference & & Reference & \\
\hline$>110$ & $0.400(0.217-0.737)$ & 0.003 & - & 0.238 & $0.397(0.207-0.761)$ & 0.005 & - & 0.290 \\
\hline
\end{tabular}

Note: $\mathrm{p}<0.05$ is indicated in bold.

Abbreviations: OS, overall survival; BMI, body mass index; PLR, platelet-lymphocyte ratio; NLR, neutrophil-lymphocyte ratio; FAR, fibrinogen-albumin ratio.

predictive ability than TNM staging in both the training and validation sets (Figure 5). Furthermore, the calibration curves showed good agreement between the nomogram predicted and actual rates for 1-year and 3-year OS and PFS both in the training and validation sets (Figures 6 and $\underline{\text { S3}}$ ).

\section{Discussion}

$\mathrm{BCa}$ is a common malignancy of the urinary tract, and the main treatments for MIBC are RC and urinary diversion reconstruction. The standard treatment for intermediaterisk and high-risk NMIBC is TURBt surgery combined 


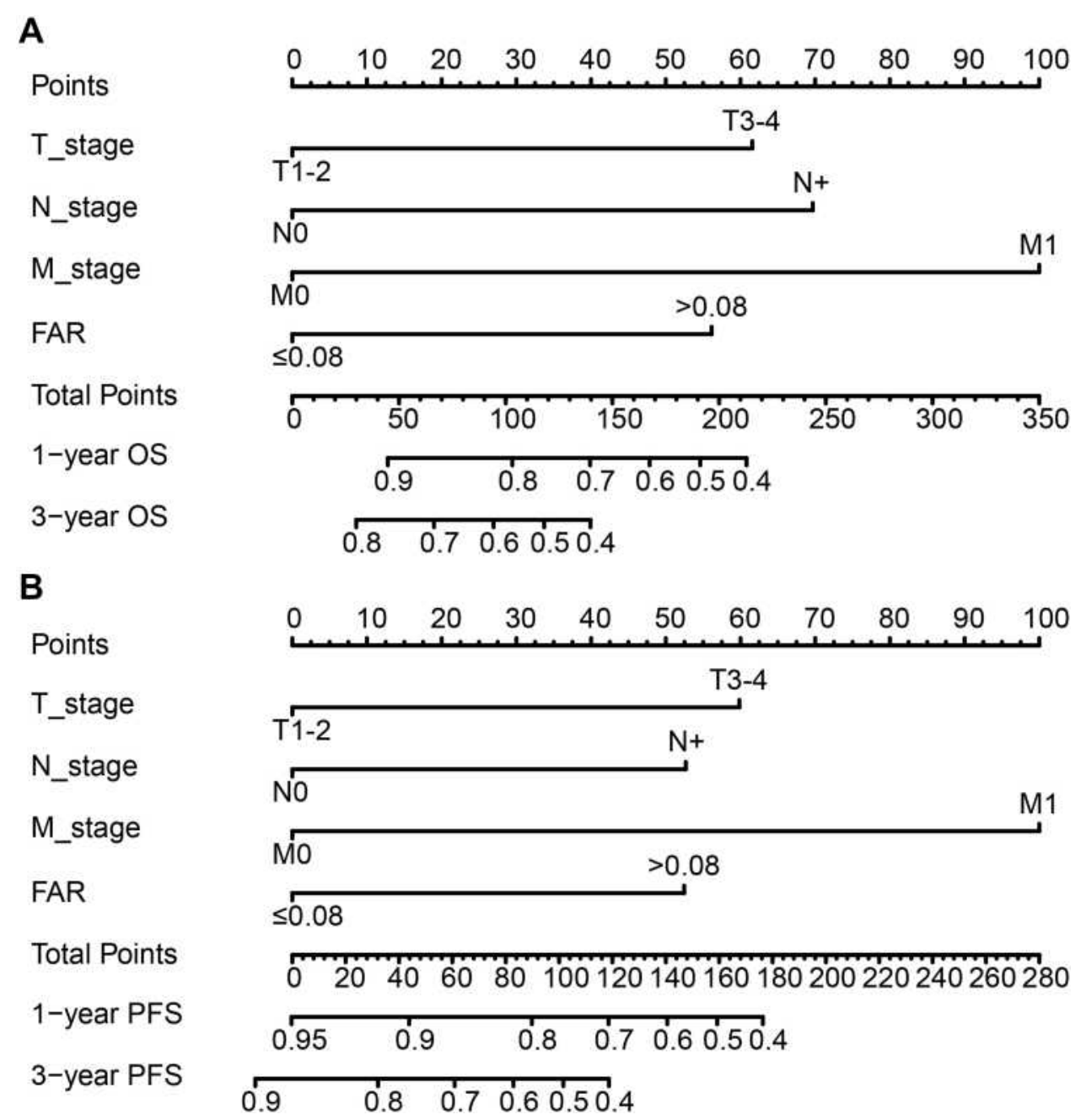

Figure 3 Nomogram predicting I-year and 3-year overall survival (OS) and progression-free survival (CSS) rate of UTUC patients. (A) OS nomogram; (B) PFS nomogram.

with drug-infused chemotherapy such as bacillus Calmette-Guérin (BCG). ${ }^{14}$ However, there is still no metabolic or inflammatory score to identify the progression of $\mathrm{BCa}$ patients or the prognostic status of $\mathrm{RC}$ patients.

To our knowledge, this is the first study to focus on the prognostic impact of preoperative FAR levels in $\mathrm{BCa}$ patients undergoing RC treatment. This study first assessed the prognostic impact of preoperative FAR in RC patients and compared it with established inflammatory biomarkers. We found that high FAR was associated with poorer prognosis and that preoperative FAR had greater predictive ability for OS and PFS than NLR and PLR in both the training and validation sets. In addition, we also constructed prognostic nomograms based on FAR in the training group and validated that this prognostic nomogram better predicted individual survival in the validation group.

Fibrinogen is a multifunctional protein that affects many cellular processes during tumorigenesis and metastasis. ${ }^{15}$ Fibrinogen, also known as coagulation factor $\mathrm{I}$, is the most abundant coagulation factor in blood produced by the liver and appears to be elevated to varying degrees in a variety of pathophysiological conditions, including surgery, infection, inflammation, trauma, and tumor. ${ }^{15,16}$ In malignant tumors, one or more coagulation abnormalities often accompany the disease. The activation of coagulation system and the release of coagulationrelated molecules play an important role in tumor 

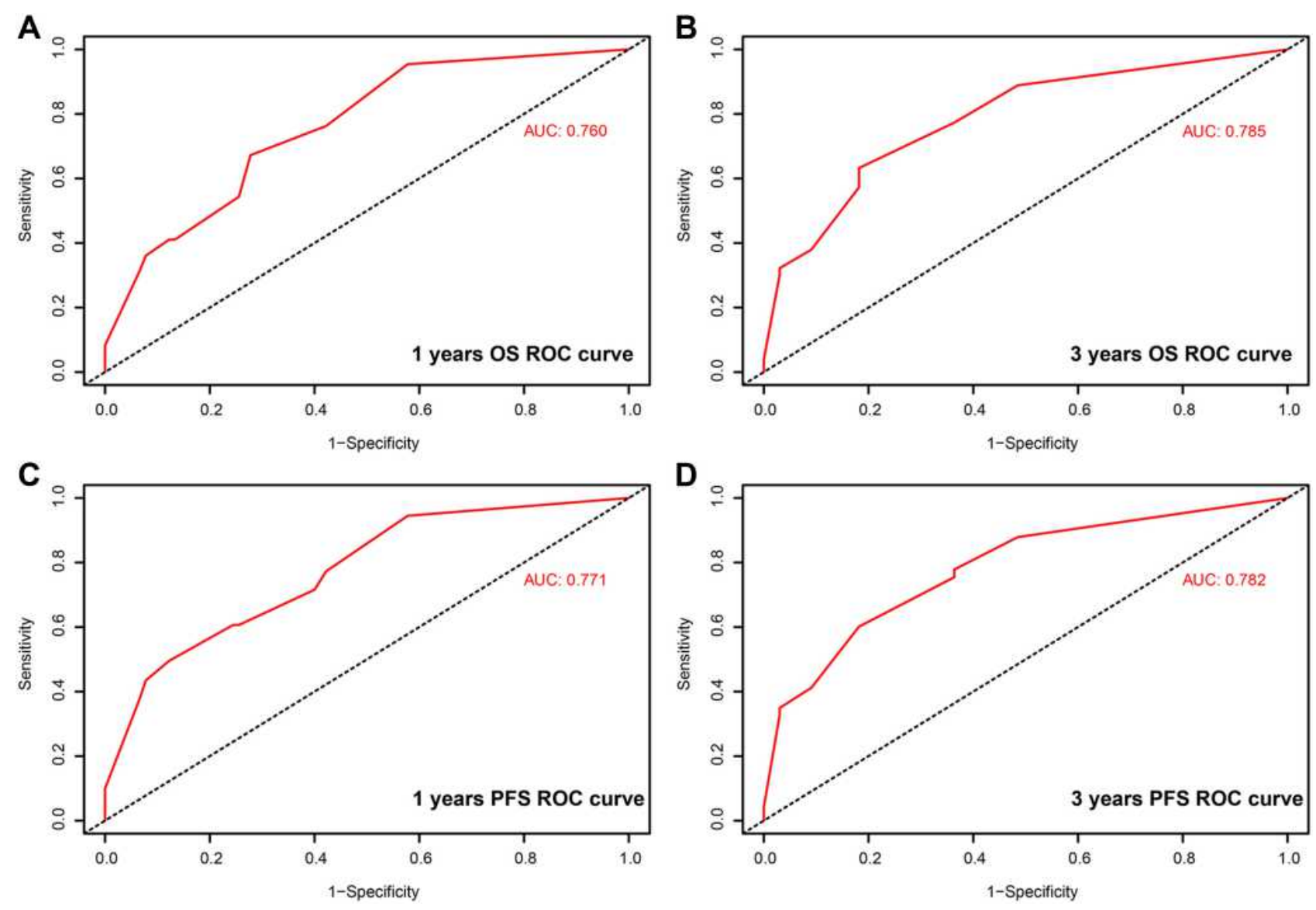

Figure 4 Receiver operating characteristic (ROC) curves for the nomogram in the training set. (A) I-year overall survival (OS) ROC curve; (B) 3-year OS ROC curve; (C) I-year progression-free survival (PFS) ROC curve; (D) 3-year PFS ROC curve.

progression, including alterations in fibrinogen content. ${ }^{17}$ A growing number of studies have found a close relationship between fibrinogen and malignancy, and its association with tumor progression, poor response to chemotherapy, and poor clinical prognosis. ${ }^{18}$ The mechanism may be that fibrinogen can play a cytoskeleton role in the extracellular matrix of tumors, thereby promoting tumor angiogenesis and enhancing tumor cell adhesion, migration and invasion. ${ }^{19}$ Fibrinogen generates proliferative signals by acting as a scaffold for binding growth factors (like FGF-2, VEGF). ${ }^{20}$ The binding of growth factors promotes cell adhesion, proliferation and migration during angiogenesis and tumor cell growth. In addition, cancer cells can synthesize and secrete additional endogenous fibrinogen, and high fibrinogen promotes IL-6 synthesis and changes the nature of leukocyte infiltration as well as stimulates $\mathrm{T}$ and $\mathrm{B}$ cells to promote chronic validation responses. ${ }^{21,22}$
Previous studies have shown that the nutritional status of tumor patients correlates with patient age, degree of disease progression and prognosis. ${ }^{23}$ Malnourished patients are often associated with low autoimmune function and can accelerate tumor cell replication. ${ }^{24}$ As one of the indexes to evaluate the preoperative nutritional status, albumin is widely used in clinic. Albumin mainly reflects the nutritional status of the body, and the deficiency of albumin in human body is closely related to the incidence of postoperative complications, secondary surgery and recurrence of malignant tumor, and albumin can be a good predictor of postoperative morbidity and mortality. ${ }^{25}$ Hypoproteinemia is not only a reliable indicator of malnutrition and cachexia in malignant tumor patients but also reflects the systemic inflammatory response of tumor patients. $^{26}$ As a new biomarker, FAR combined with blood nutritional status and blood coagulation function has been widely used to predict the prognosis of various malignant tumors. ${ }^{8-10}$ 

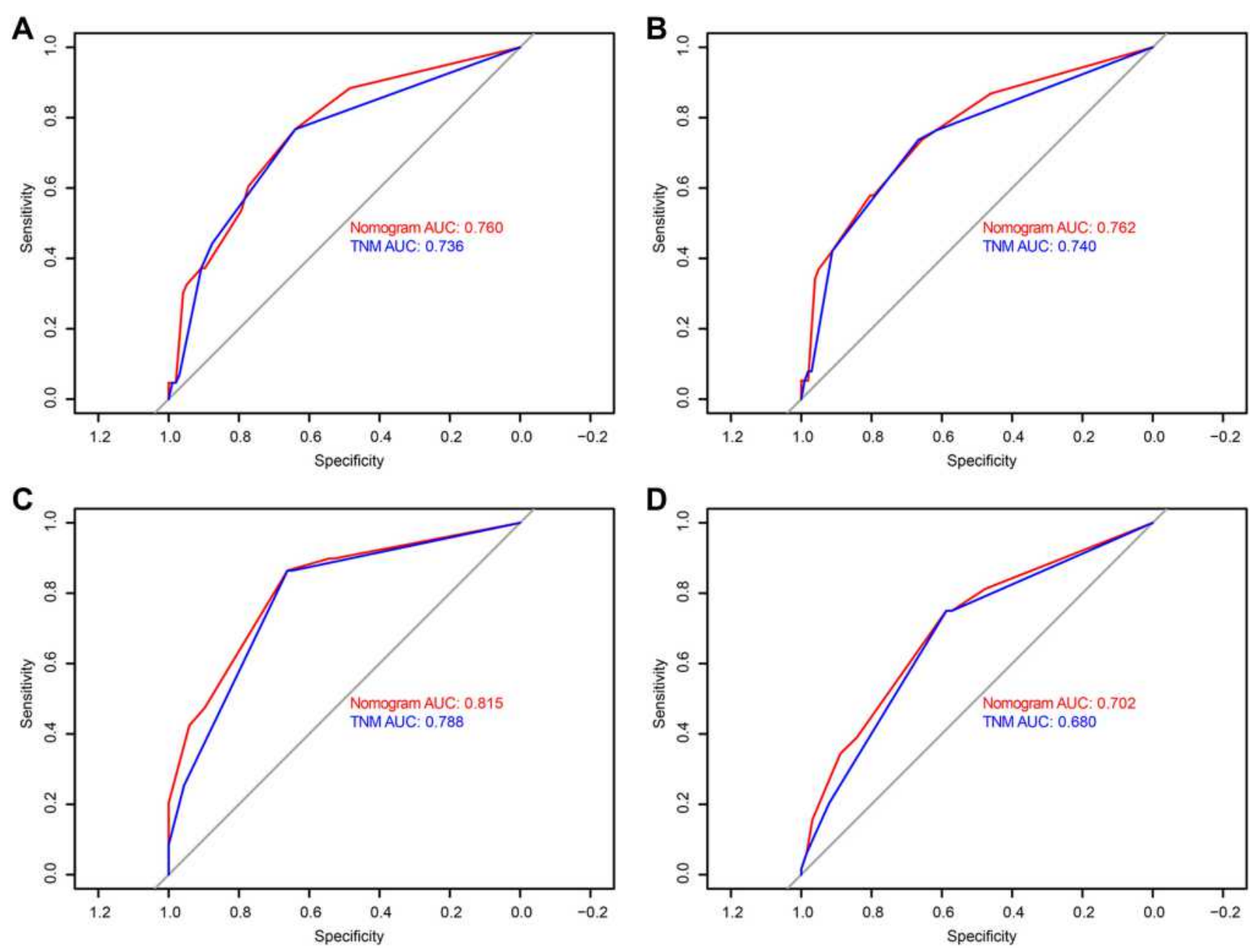

Figure 5 Receiver operating characteristic (ROC) curves shows the predictive ability. (A) ROC for the overall survival (OS) in the training set; (B) ROC for the progression-free survival (PFS) in the training set; (C) ROC for the overall survival (OS) in the validation set; (D) ROC for the progression-free survival (PFS) in the validation set.

PLR and NLR are commonly used indicators of systemic inflammation. Studies have shown that NLR and PLR can be used as independent risk factors for patients with $\mathrm{BCa}{ }^{27,28}$ In this study, we first determined the optimal cutoff value of FAR using X-tile and divided the patients into high FAR $(>0.08)$ group and low FAR $(\leq 0.08)$ group. We found that FAR was associated with sex, T stage, N stage, NLR and hemoglobin. We compared FAR with NLR and PLR and found that FAR had better ability to predict OS and PFS than PLR and NLR in both the training and validation sets. In addition, multivariate Cox regression analysis showed that FAR was an independent prognostic factor for OS and PFS, and high FAR was associated with poor prognosis (OS: HR, 2.031, 95\% $\mathrm{CI}=1.071-3.851 ; \quad \mathrm{P}=0.030 ; \quad$ PFS: $\mathrm{HR}, \quad 2.330, \quad 95 \%$ $\mathrm{CI}=1.160-4.680 ; \mathrm{P}=0.017)$. Moreover, we also constructed a prognostic nomogram including FAR and found that the constructed nomogram could better predict 1-year and 3-year OS and PFS in BCa patients treated with RC.

This study had some limitations. This is a retrospective study, which may also lead to bias in data selection and analysis. Although it is a two-center study, the sample size is still small and a prospective large sample study is needed. Despite these limitations, this study is the first to reveal that high $\mathrm{FPR}$ predicts poor prognosis in $\mathrm{BCa}$ patient treatment with RC.

\section{Conclusion}

In summary, we found preoperative high FAR is a poor prognostic factor for OS and PFS in BCa patients treated with $\mathrm{RC}$, and a prognostic nomogram based on FAR can better predict individual survival. 

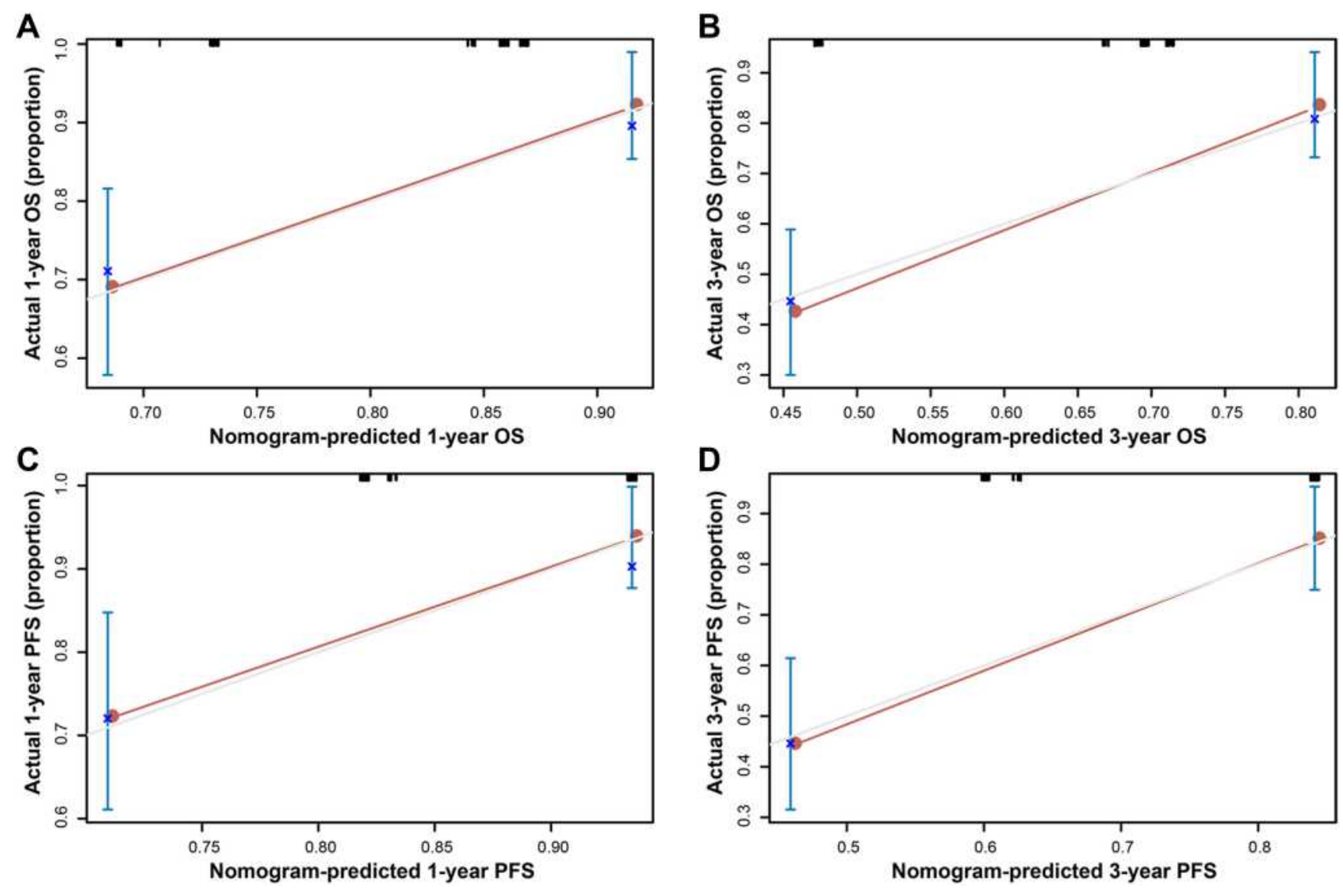

Figure 6 Calibration plot of the nomogram in the training set. (A) I-year overall survival (OS); (B) 3-year OS; (C) I-year progression-free survival (PFS); (D) 3-year PFS.

\section{Data Sharing Statement}

The dataset used during the study is available from the corresponding author on a reasonable request.

\section{Ethics Approval and Consent to Participate}

The methodology of this study followed the criteria outlined in the Declaration of Helsinki (as revised in 2013) and was ethically approved by the Ethics Committee Review Board of Xuzhou Central Hospital (No. 2019XZSY012) and Ethics Committee Review Board of The Second Affiliated Hospital of Nantong University (No. 2018-027).

\section{Acknowledgment}

We thank all the researchers and study participants for their contributions.

\section{Author Contributions}

All authors made a significant contribution to the work reported, whether that is in the conception, study design, execution, acquisition of data, analysis and interpretation, or in all these areas; took part in drafting, revising or critically reviewing the article; gave final approval of the version to be published; have agreed on the journal to which the article has been submitted; and agree to be accountable for all aspects of the work.

\section{Funding}

The present study was supported by The Jiangsu Province, Natural Science Research Projects (17KJB360001, 18KJB180007), The Nantong Science and Technology Program (MS12020025).

\section{Disclosure}

The authors declare that they have no competing interests.

\section{References}

1. Bray F, Ferlay J, Soerjomataram I, Siegel RL, Torre LA, Jemal A. Global cancer statistics 2018: GLOBOCAN estimates of incidence and mortality worldwide for 36 cancers in 185 countries. $C A$ Cancer J Clin. 2018;68(6):394-424. doi:10.3322/caac.21492 
2. Czerniak B, Dinney C, McConkey D. Origins of bladder cancer. Annu Rev Pathol. 2016;11(1):149-174. doi:10.1146/annurev-pathol $-012513-104703$

3. Babjuk M, Bohle A, Burger M, et al. EAU guidelines on non-muscleinvasive urothelial carcinoma of the bladder: update 2016. Eur Urol. 2017;71(3):447-461. doi:10.1016/j.eururo.2016.05.041

4. Mao W, Huang X, Wang L, et al. Circular RNA hsa_circ_0068871 regulates FGFR3 expression and activates STAT3 by targeting miR-181a-5p to promote bladder cancer progression. J Exp Clin Cancer Res. 2019;38(1):169. doi:10.1186/s13046-019-1136-9

5. Jain A, Zhang Q, Toh HC. Awakening immunity against cancer: a 2017 primer for clinicians. Chin J Cancer. 2017;36(1):67. doi:10.1186/s40880-017-0233-4

6. Weisel JW. Fibrinogen and fibrin. Adv Protein Chem. 2005;70:247-299.

7. Seo MH, Choa M, You JS, et al. Hypoalbuminemia, low base excess values, and tachypnea predict 28-day mortality in severe sepsis and septic shock patients in the emergency department. Yonsei Med J. 2016;57(6):1361-1369. doi:10.3349/ymj.2016.57.6.1361

8. Tan Z, Zhang M, Han Q, et al. A novel blood tool of cancer prognosis in esophageal squamous cell carcinoma: the fibrinogen/albumin ratio. $J$ Cancer. 2017;8(6):1025-1029. doi:10.7150/jca.16491

9. Xu Q, Yan Y, Gu S, et al. A novel inflammation-based prognostic score: the fibrinogen/albumin ratio predicts prognoses of patients after curative resection for hepatocellular carcinoma. J Immunol Res. 2018;2018:4925498. doi:10.1155/2018/4925498

10. Liu J, Gan Y, Song H, Zhu K, Zhang Q. The predictive value of the preoperative fibrinogen-albumin ratio on the postoperative prognosis of renal cell carcinoma. Transl Androl Urol. 2020;9(3):1053-1061. doi: $10.21037 /$ tau-19-873

11. Park JH, Moon KC. Tumor, Nodes, Metastases (TNM) Classification System for Bladder Cancer. 2018:181-184.

12. Enquselassie F. 50th anniversary of declaration of Helsinki. Ethiop Med J. 2014;52(1):2p preceding 1.

13. Proctor MJ, Horgan PG, Talwar D, Fletcher CD, Morrison DS, McMillan DC. Optimization of the systemic inflammation-based Glasgow prognostic score: a Glasgow Inflammation Outcome Study. Cancer. 2013;119(12):2325-2332. doi:10.1002/cncr.28018

14. Ferro M, Katalin MO, Buonerba C, et al. Type 2 diabetes mellitus predicts worse outcomes in patients with high-grade $\mathrm{T} 1$ bladder cancer receiving bacillus Calmette-Guerin after transurethral resection of the bladder tumor. Urol Oncol. 2020;38(5):459-464. doi:10.1016/j.urolonc.2020.02.016

15. Bratek-Skicki A, Zeliszewska P, Ruso JM. Fibrinogen: a journey into biotechnology. Soft Matter. 2016;12(42):8639-8653. doi:10.1039/ C6SM01895E

16. Pieters M, Wolberg AS. Fibrinogen and fibrin: an illustrated review. Res Pract Thromb Haemost. 2019;3(2):161-172. doi:10.1002/ rth2.12191
17. Lee SY, Song CW, Levitt SH. Change in fibrinogen turnover in tumors by hyperthermia. Eur J Cancer Clin Oncol. 1985;21 (12):1507-1513. doi:10.1016/0277-5379(85)90246-9

18. Kwaan HC, Lindholm PF. Fibrin and fibrinolysis in cancer. Semin Thromb Hemost. 2019;45(4):413-422. doi:10.1055/s-0039-1688495

19. Li S, Lan X, Gao H, et al. Systemic Inflammation Response Index (SIRI), cancer stem cells and survival of localised gastric adenocarcinoma after curative resection. J Cancer Res Clin Oncol. 2017;143 (12):2455-2468. doi:10.1007/s00432-017-2506-3

20. Mosesson MW. Fibrinogen and fibrin structure and functions. $J$ Thromb Haemost. 2005;3(8):1894-1904. doi:10.1111/j.15387836.2005.01365.x

21. Sahni A, Khorana AA, Baggs RB, Peng H, Francis CW. FGF-2 binding to fibrin(ogen) is required for augmented angiogenesis. Blood. 2006;107(1):126-131. doi:10.1182/blood-2005-06-2460

22. Ridker PM, Howard CP, Walter V, et al. Effects of interleukin-1beta inhibition with canakinumab on hemoglobin A1c, lipids, C-reactive protein, interleukin- 6 , and fibrinogen: a phase IIb randomized, placebo-controlled trial. Circulation. 2012;126(23):2739-2748. doi:10.1161/CIRCULATIONAHA.112.122556

23. Mantzorou M, Koutelidakis A, Theocharis S, Giaginis C. Clinical value of nutritional status in cancer: what is its impact and how it affects disease progression and prognosis? Nutr Cancer. 2017;69 (8):1151-1176. doi:10.1080/01635581.2017.1367947

24. Virizuela JA, Camblor-Alvarez M, Luengo-Perez LM, et al. Nutritional support and parenteral nutrition in cancer patients: an expert consensus report. Clin Transl Oncol. 2018;20(5):619-629. doi:10.1007/s12094-017-1757-4

25. Jones $\mathrm{CH}$, Akbani H, Croft DC, Worth DP. The relationship between serum albumin and hydration status in hemodialysis patients. $J$ Ren Nutr. 2002;12(4):209-212. doi:10.1053/jren.2002.35295

26. Watt DG, McSorley ST, Park JH, Horgan PG, McMillan DC. A postoperative systemic inflammation score predicts short- and long-term outcomes in patients undergoing surgery for colorectal cancer. Ann Surg Oncol. 2017;24(4):1100-1109. doi:10.1245/s10434016-5659-4

27. Vartolomei MD, Ferro M, Cantiello F, et al. Validation of neutrophil-to-lymphocyte ratio in a multi-institutional cohort of patients with T1G3 non-muscle-invasive bladder cancer. Clin Genitourin Cancer. 2018;16(6):445-452. doi:10.1016/j. clgc.2018.07.003

28. Ferro M, Di Lorenzo G, Vartolomei MD, et al. Absolute basophil count is associated with time to recurrence in patients with high-grade $\mathrm{T} 1$ bladder cancer receiving bacillus Calmette-Guerin after transurethral resection of the bladder tumor. World $J$ Urol. 2020;38(1):143-150. doi:10.1007/s00345-019-02754-2

\section{Publish your work in this journal}

Cancer Management and Research is an international, peer-reviewed open access journal focusing on cancer research and the optimal use of preventative and integrated treatment interventions to achieve improved outcomes, enhanced survival and quality of life for the cancer patient.
The manuscript management system is completely online and includes a very quick and fair peer-review system, which is all easy to use. Visit http://www.dovepress.com/testimonials.php to read real quotes from published authors. 